\title{
Implementation of Automatic Gate Control for Railroad Switch and Anti-Collision System using Arduino
}

\author{
Mahesh Kumar A S, Rajesh A S, Bhanu H S
}

\begin{abstract}
India one of the most train transportation adaptable country. Most of accidents occur at while crossing, due to manual operation or negligence of people. Train accidents are going on based on railway gate controlling, track switching system, collision system and protocols using for communication. Head-on collisions are the most problematic issues because of manual switching process and collision on railway tracks. The accidents results to significant property damage of organizations, financial losses and individual's losses. Every individual life is very important. This paper presents an Implementation of Automatic Gate Control for Railroad Switch and Anti-Collision System using Arduino; it majorly avoids accidents while crossing and provides switching techniques for railroad. Anti-collision systems sense the obstacles present on track within a specific distance and immediately alert the pilot which avoids the train accidents.
\end{abstract}

Keywords-: Global Positioning System [GPS], Liquid Crystal Display [LCD], Infrared sensor [IR], Ultrasonic sensor [UV], Radio Frequency Identification [RFID].

\section{INTRODUCTION}

More populated country like India railway is most convenient system for travelling. The major role of the railways is to serve the public in transportation and for carrying the tones of goods every day. Railways provide a best alternative to other transport medium in case of energy efficient. As a result, the number of people using it increases day by day from last few decades. Hence it plays a major role in changing Indian economy and one of the more revenue departments.

First railway proposals planned in during at 1832 was made in Madras. From the relative literature surveys, it was organized by Indian Railway Department and around 17 percentage of railway accidents occurred while crossing [6]. Accidents of which majority occurs at passive railway crossings. The conventional method of using for generation of signals and operating systems makes sometime false decision which causes major problems to individuals and organizations. The below line graph shows the Indian railway accidents form 2010- 2018.

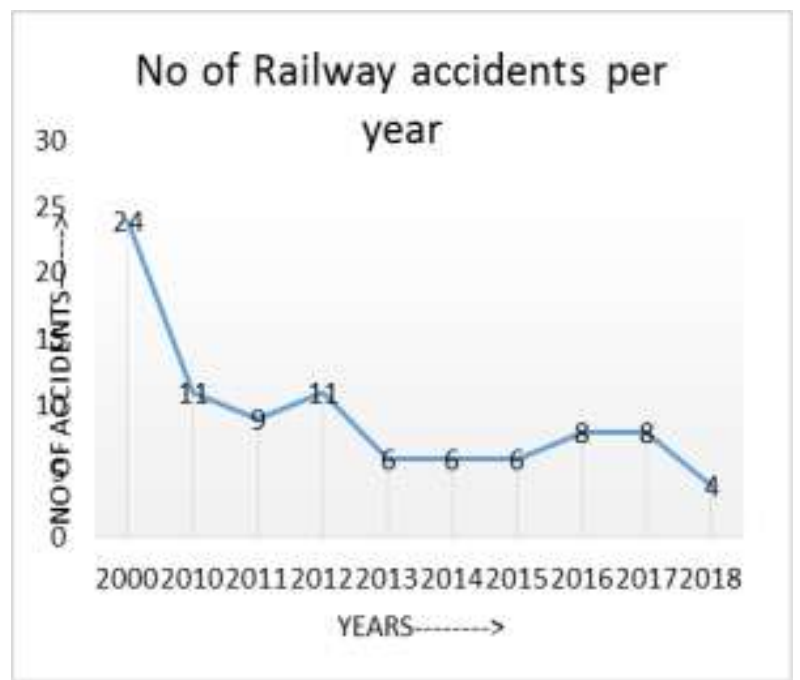

Fig 1: Number of Railway accidents per year

From the Fig 1 it is clear about the accidents are more in the beginning due to carelessness of road users, due to manual errors or due to obstacles on the track, accidents are happening. Therefore, to reduce these accidents care must be taken because human or animals lives are precious and decrease the impact on nation's economy is also important. It's railway management's responsibility to make the train journey safe and reliable.

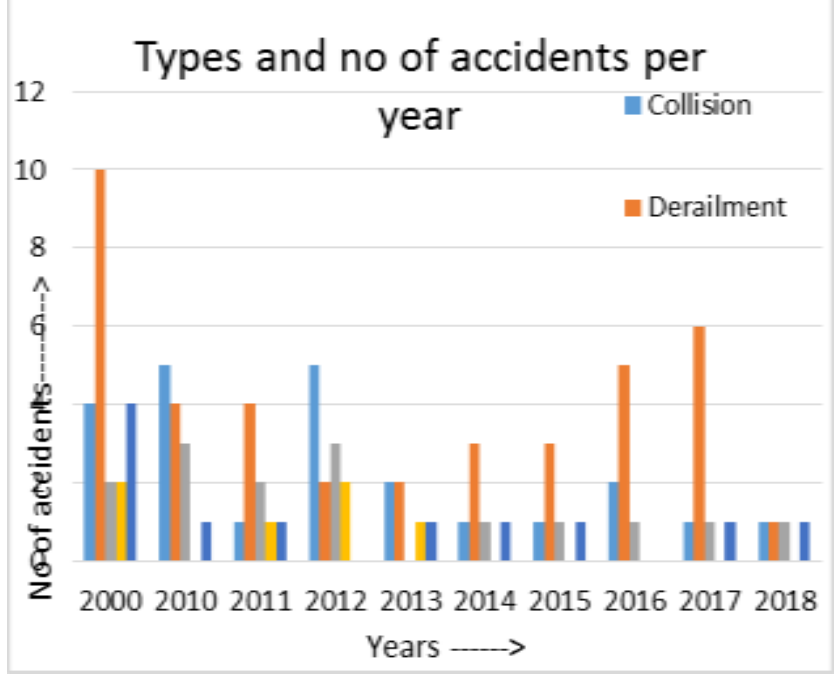

Fig 2: Types and Number of accidents per year 


\section{IMPLEMENTATION OF AUTOMATIC GATE CONTROL FOR RAILROAD SWITCH AND ANTI-COLLISION SYSTEM USING ARDUINO}

\section{A. Objectives}

This paper describes controlling method of railway gates accurately by automation technique at crossing, and making use of sensors and actuators. Main sensors and actuators used in this paper are notably IR sensors or ultrasonic sensor and buzzers, LED display, servo motors respectively. Automatic method of switching railroad using RFID and servo motors almost minimized errors obtained by manual track switching. Most of the train accidents are due to of obstacles like animals, vehicles passing, and other things, those are present on the railway track. The above mentioned problems are solved by Anti-collision system using ultrasonic sensor and motor, which gives the information to the pilot.

\section{B. Literature Survey}

\section{i. Based on Railway Gate}

M. Duraishanmugapriyan et al [1] introduce an Automatic technique for Railway Gate Monitoring and Controlling System, it use microcontroller ATmega328P along with sensors. The system uses IR sensors mainly to find arrival and departure timings of the train in station. For the transmission of sensor output information to controller at remote areas RF transmitter and receiver are used. Microcontroller main unit of system, it plays a vital role by receiving input signal from the all sensors and sends the information to the gate motor driver for opening and closing the gate. The output signal Arduino will active LCD display and alarm.

Dhanashree Anant Umbarkar et al [2] Presented PLC Based system which is fully automated technique in railways [2]. Technique includes self-acting PLC system which gives information about train collision at crosses.

KarthikKrishnamurthi et al [3] developed the concept of automatic control of railroad using sensors. Infra-red sensors are used to find arrival and departure timings of trains at the railway level crossing. Opening and closing of gates are controlled by Arduino at level.

Acy M. Kottalil et al [4] developed the method of Automatic Gate Controlling System in Railways [4].This paper uses ATmega 16A microcontroller and IR Transceiver. This type of system decreases the time needed for closing gates.

ii. Based on Track switching System Based on Collision System Based on Protocols used for communication

Khizir Mahmud Md. Reya Shad Azim, et al [5] implemented the concept of Automatic Switching of train track System using computerized organization. The system was designed to control railway track including railway switches and signals within a given area from a single point. The track switching system implemented with microcontroller, sensor, visual basic, serial communication, and low power DC motor. Photodiode is used for detecting IR radiation which ensures a reliable detection of train's entrance.

iii. Based on Collision System Based on Protocols used for communication

Dr. K.R.R. Mohan Raoet al [6] developed the concept of accident avoidance using Arm7. This system also uses
ZigBee protocol, buzzers, ARM7LPC2148, LCD display, MAX232 to provide safety measure for accident of trains.

RanuDewanganetsl [7] introduced the method of controlling accident on Railway Tracks automatically. Main contribution of paper is it finds the obstacle on the tract within specified distance by setting time.

Dogan Ibrahim et al [8] present a new concept called as Smart Train Collision Detection System using a Microcontrollers. Implemented systems include microcontroller, RFID, GPS and an RF transmitter/receiver module to detect probable collisions and soon intimation to drivers, if the train present in the track.

Naga HemaKumari.V et al [9] implemented a new method for avoiding train accident by using sensors, this paper explain how accidents are reduced and provides full safety to people by developing module using LPC2184 processor, UV sensor, IR sensor. Head-on and rear end collision can be achieved. Radio modems are used for communication by the anti-collision device.

\section{Iv. Based on Protocols used for communication}

Abid Rahim et al [10] introduced a concept using low cost wireless sensor accident of train is reduced. Also this method uses Arduino RF24L01. This system developed with lot of flexibility and also cheaper compared to all other existing systems. Main feature is capable of handling, both sensors and sensor nodes.

\section{METHODOLOGY}

\section{a. Railway gate controlling system}

Railway safety is the most important concern in railways. Railways transportation considers being more comfortable with cheapest fair. Most of the accidents are due to carelessness and manual operations of the systems. Hence there is a need of automatic railway crossing controller.

\section{b. an automatic railway gate controlling system}

In general, Gate keeper manually operates the railway gates at level crossing. When the train starts to leave the station, the station in-charge delivers this information to the closest gatekeeper to get ready. In situations where the train gets delayed, the gates remain closed for long durations causing dense traffic jam at the level crossings. The rate of manual error that could occur at these level crossings are high because they are unsafe to perform without actual knowledge about the train time table. These human interventions can be avoided by automating the process and it doesn't degrade the existing safety level 


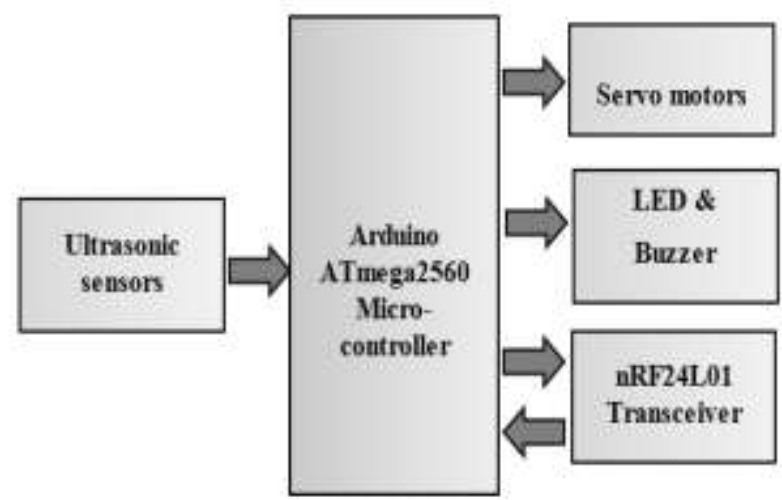

Fig 3: Block diagram of gate controlling system

A block diagram of an automatic controlling of railway system is shown in Fig 3. By using this system at the level crossing the arrival or departure can be determine using Ultrasonic sensor. The opening or closing of the level crossing gates automatically with the help of microcontroller and servo motors. Warning signals are given near roads using Buzzer sound.

c. Working of an automatic railway gate controlling system

The ultrasonic sensors are used on sides of gate at about $4 \mathrm{~km}$ from crossing. As soon as train arrival is detected, the detected signal is sent to microcontroller. Based on that signal, the microcontroller will turn on buzzer for warning, the road user knows the train arrival and sends the actuation information for closing the gate to the servo motors.

After closing gate, the nRF24L01 transceiver broadcast the message of 'Gate closed' to the near control room and to the loco-pilot in the train. But there may be a chance that during this automation process, a vehicle may be locked between the crossing gates. At this situation, the obstacle between the crossing gates could be detected with the help of ultrasonic sensor and it will be intimated to the loco-pilot in the train as well as nearest control room through transceiver earlier than the train arrives $3 \mathrm{~km}$ from the railway gate. Thus, the man power could be reduced and at the same time accidents at level crossings can be avoided into maximum extent.

If no obstacle is sensed between the crossing gates, the train passes as usual. Another sensor which is on either side of gate detects the departure of the train, the controller receives the detected signal and in turns it sends the actuation signal for opening the gate. After opening the gate, the transceiver sends the message of 'Gate opened', to the control room and loco-pilot. This enables the real time communication between the gate controlling system and loco-pilot as well as nearest control room. The broadcasting of information about the gate status and controlling action enables them to take immediate action, when any fault is detected or any obstacles is detected in-between the crossing gate. This automatic gate controlling system may reduce the accidents, especially accidents at railway level crossing. d. Working flow of an automatic gate controlling system

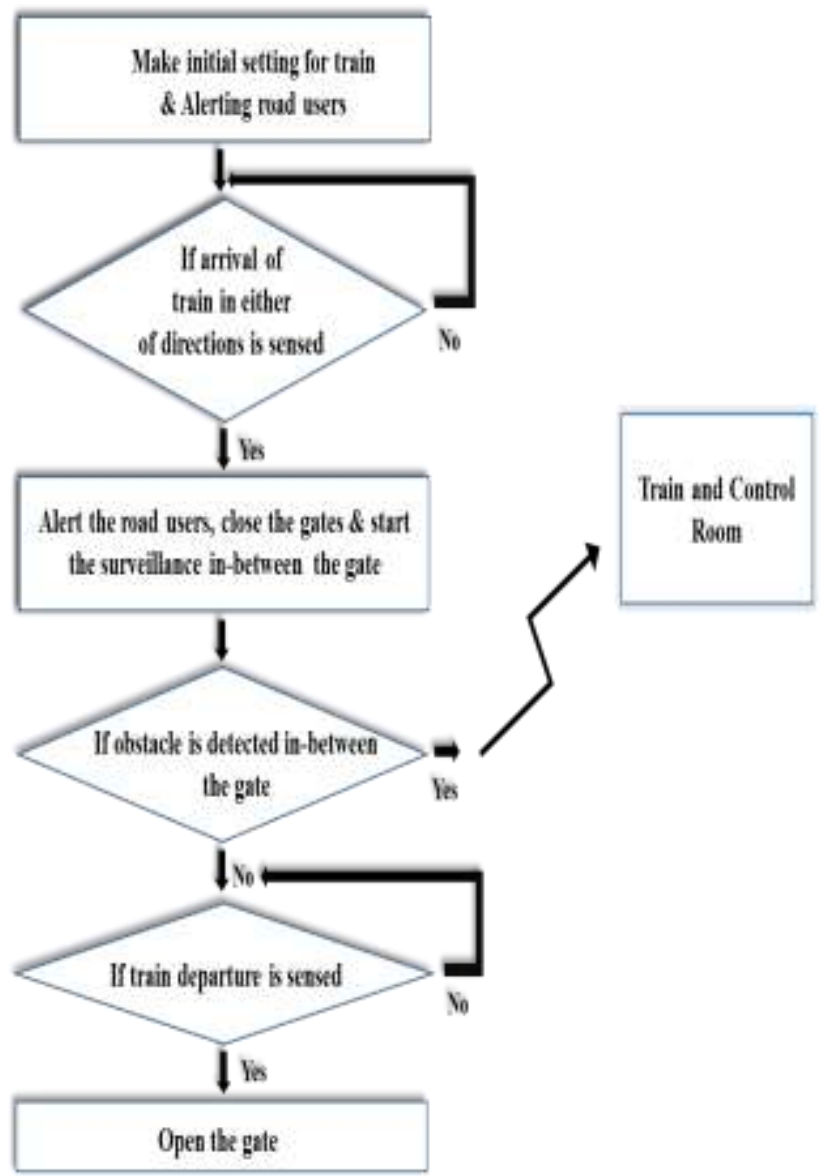

Fig 4: Working flow of an automatic gate controlling system

The flow chart gives the working flow of the system. Fig 4: shows the flow chart for an automatic railway gate controlling system. Let's discuss the working flow using by following steps.

Assuming that initially the gate is opened and people are passing through level crossing.

Step 1: Make initial setting to alert for train and people via LED indication.

Step 2: As soon as train comes in each of two sides is sensed by sensors, the sensed signal is transferred to microcontroller.

Based on that signal, the microcontroller will turn on buzzer for warning the road user about the train arrival and sends the actuation signal to the servo motors. If not the signal is again sensed by the ultrasonic sensor.

Step 3: Ultrasonic sensor detects the obstacle in between the gate and will intimates to the loco-pilot in the train as well as nearest control room through nRF24L01 transceiver before the train arrival. If no obstacle is sensed by the ultrasonic sensor between the crossing gates, the train passes as usual.

Step 4: Another ultrasonic sensor is used on other side of gate, senses the moving of train from station, then controller receives the sensed signal and in turns it sends the actuation signal for opening the gate. 


\section{IMPLEMENTATION OF AUTOMATIC GATE CONTROL FOR RAILROAD SWITCH AND ANTI-COLLISION SYSTEM USING ARDUINO}

e. Block diagram of Railroad switching system

At present the Indian railway system are using manual track switching system where switching the track manually using hand lever. When a train confirms it's incoming, a person or labor has to go there and pull the lever to switch the track.

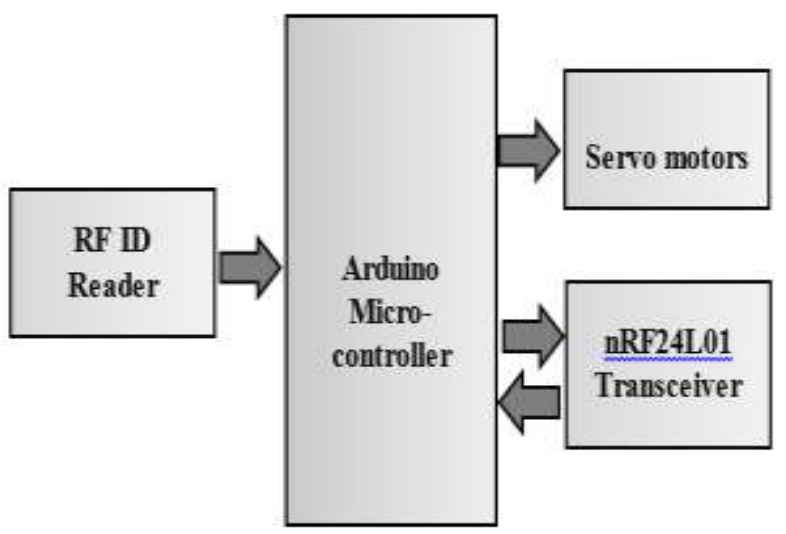

Fig 5: Railroad switching system

By this manual switching, chances of errors are more. By improper track switching, two trains will enter into same track which causes head-on or rare-end collision of two trains. To avoid this kind of errors in manual switching an automatic railroad switching system is proposed. This automatic system switches the track based on unique ID of the train.

\section{$f$. Working of an automatic railroad switching system}

The ID-tags is mounted on the train and the RFID Reader is fixed on the side of the track. The reader retrieves the unique ID information of the train.

Based on unique ID of train, the railway track changes using servo motor and it transfer the information to the pilot as well as the nearest control room at a time using the nRF24L01 transceiver. By this system it reduced the man power and also reduces the manual errors done by humans.

\section{g. Flow chart of Railroad switching system}

The flow chart gives the working flow of the system. Fig 3.4 shows the flow chart for a Railroad switching system. Let's discuss the working flow using by following steps.

Step 1: ID-tags is mounted on the train \& RFID reader situated at some range of distance from switching system senses the Radio Frequency Identification number.

Step 2: So that the RFID reader retrieves the unique ID information of the train.

Step 3: With the help of unique ID of train, the railway track changes using servo motor and it transfer the information to the pilot as well as the nearest control room at a time using the nRF24L01 transceiver.

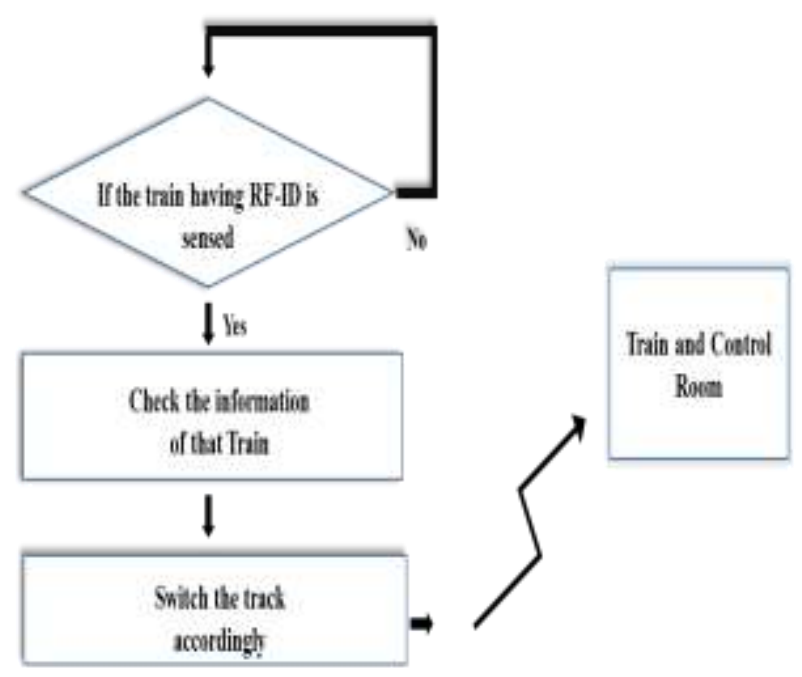

Fig 6: Flow chart of Railroad switching system

\section{h. Anti-collision system}

Nowadays train accidents are common in forest region due to the animals or any obstacles on the train. Currently there is no anti-collision system to avoid the train accidents or train collisions with obstacles or animals in the forest area or an accidental zone.

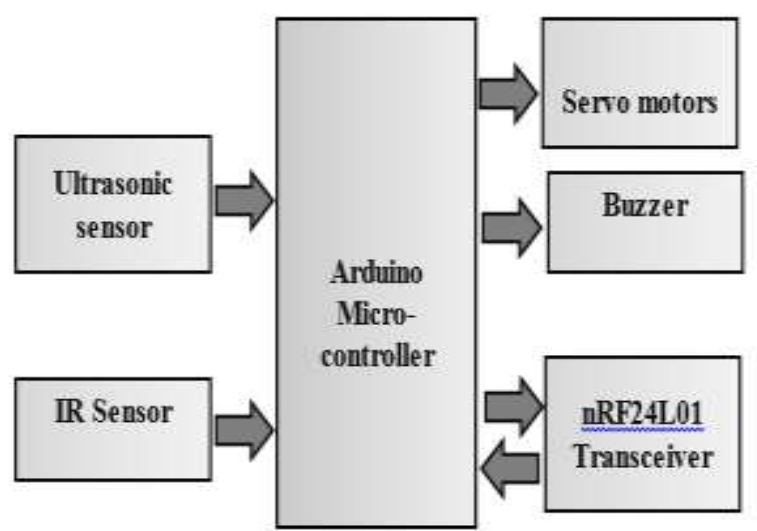

Fig 7: Block diagram of Anti-collision system

Due to accidents on the track, there are so many animals are killed or crushed under the train. Even accidents may happen because of humans on the track. This leads to loss of lives and it also leads to delay of train. These kinds of accidents also impact on economy of nation. Hence, the soul idea was proposed to reduce these kinds of accidents by implementing anti-collision system beside the track, which sense for the obstacle on the track. If obstacle is present on track, the system send the details to the nearest control room as well as loco-pilot on the train.

\section{j. Working of an anti-collision system}

IR sensor is placed at $3 \mathrm{~km}$ from the anti-collision system, once IR sensor senses the coming of the train, it transfer the information to the controller to activate the anti-collision system.

An anti-collision system is made using ultrasonic sensor and servo motor, where ultrasonic sensor senses the obstacles 
on the track and the servo motor is used to rotate the ultrasonic sensor for 0to 180 degree. So that the ultrasonic sensor can cover wide range of track for sensing the obstacles or any animals or humans on the track. This system helps to sense the obstacles on the track.

If the obstacle is present on the track it will send the information about the obstacle on the track using nRF24L01 transceiver, to the present train on that track as well as nearest control room. This kind of broadcasting the information helps them to take immediate action to avoid accidents or collisions on the track.

Anti-collision systems are especially useful as it automatically alerts about the obstacles on the track. By this anti-collision system chances of train collisions and accidents on railway track may reduce. It also saves the lives of animals in forest region or any accidental zones.

\section{l. flow of Anti-collision system}

The flow chart gives the working flow of the system. Fig 3.6 shows the working flow for a Anti-collision system. There is a detailed working flow by using following steps.
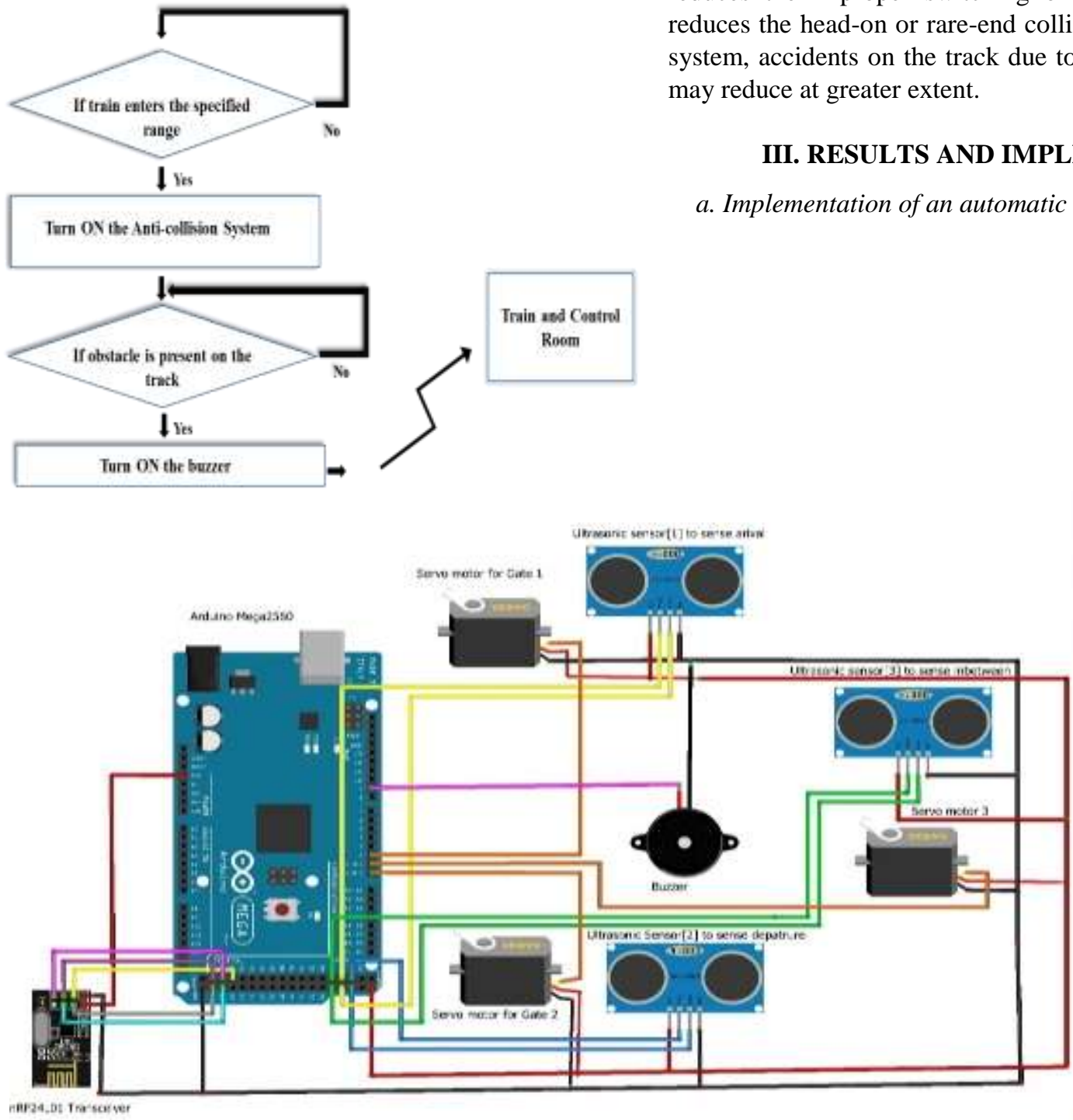

Fig 9: Implementation of an automatic railway gate controlling system may reduce at greater extent.
Fig 8: Flow of Anti-collision system

Step 1: If train enters the specified range turns on the anti-collision system via LED indication.

Step 2: If the obstacles present on railway track. Ultrasonic sensor senses for the obstacles on the track and to rotate the ultrasonic sensor, servo motor is used for 0 to 180 degrees. So that the ultrasonic sensor can cover wide range of track for sensing the obstacles or any animals or humans on the track.

Step 3: After detecting the obstacle using ultrasonic sensor, it will turn on the buzzer immediately and automatically sends information to the nearest control room via nRF24L01 transceiver.

By adapting automatic gate controlling method, accidents at crossing may reduce and also reduces the man power needed for opening and closing of railway gates. It also reduces the time for closing gate and also reduces the manual errors done by the humans.

Similarly, by employing the automatic railroad switching system, manual errors done by humans can be reduce. It also reduces the improper switching of track, which in turns reduces the head-on or rare-end collisions. By anti-collision system, accidents on the track due to obstacles on the track

\section{RESULTS AND IMPLEMENTATION}

a. Implementation of an automatic gate controlling system 


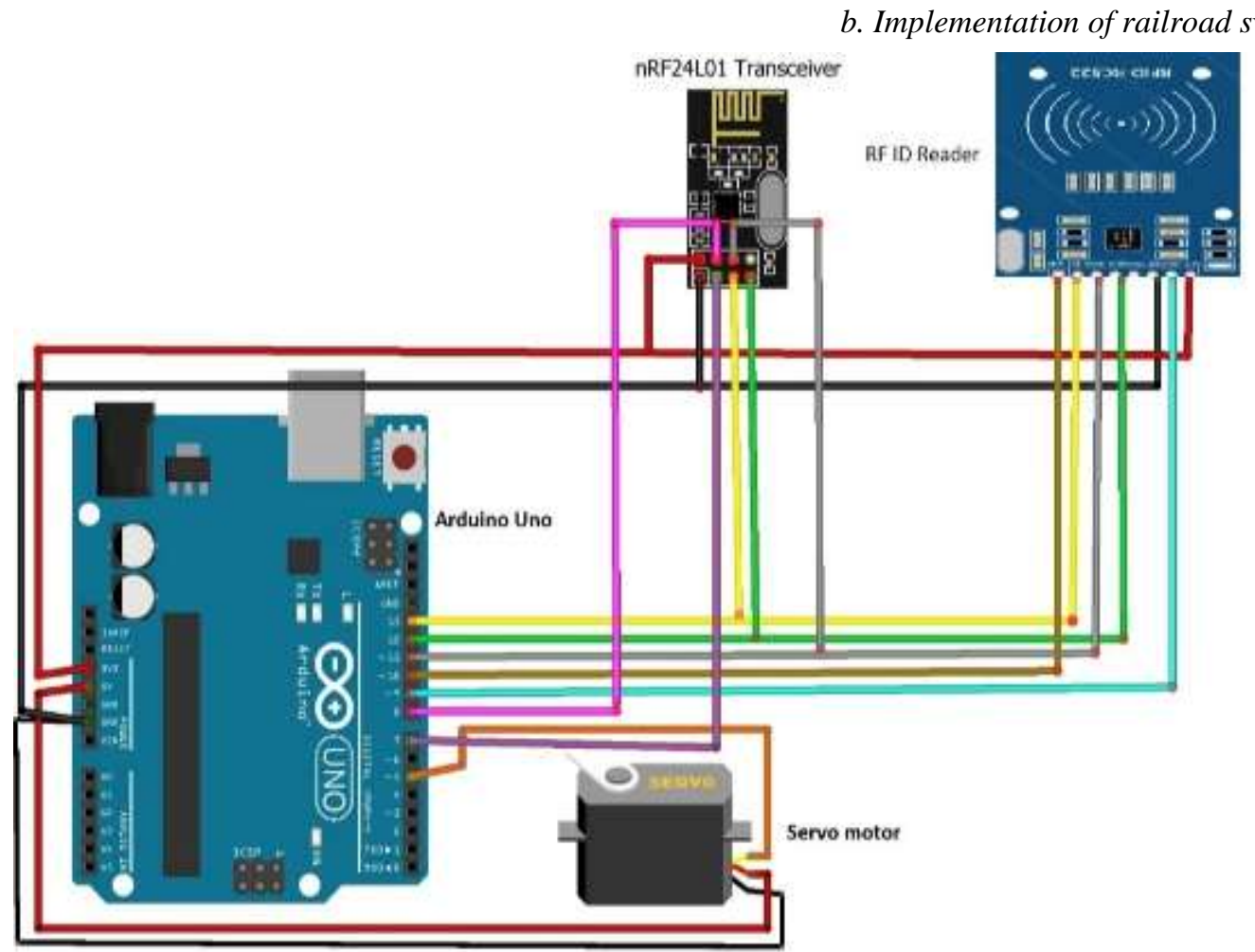

Fig 10: Implementation of railroad switching system

fritzing

c. Implementation of an anti-collision system

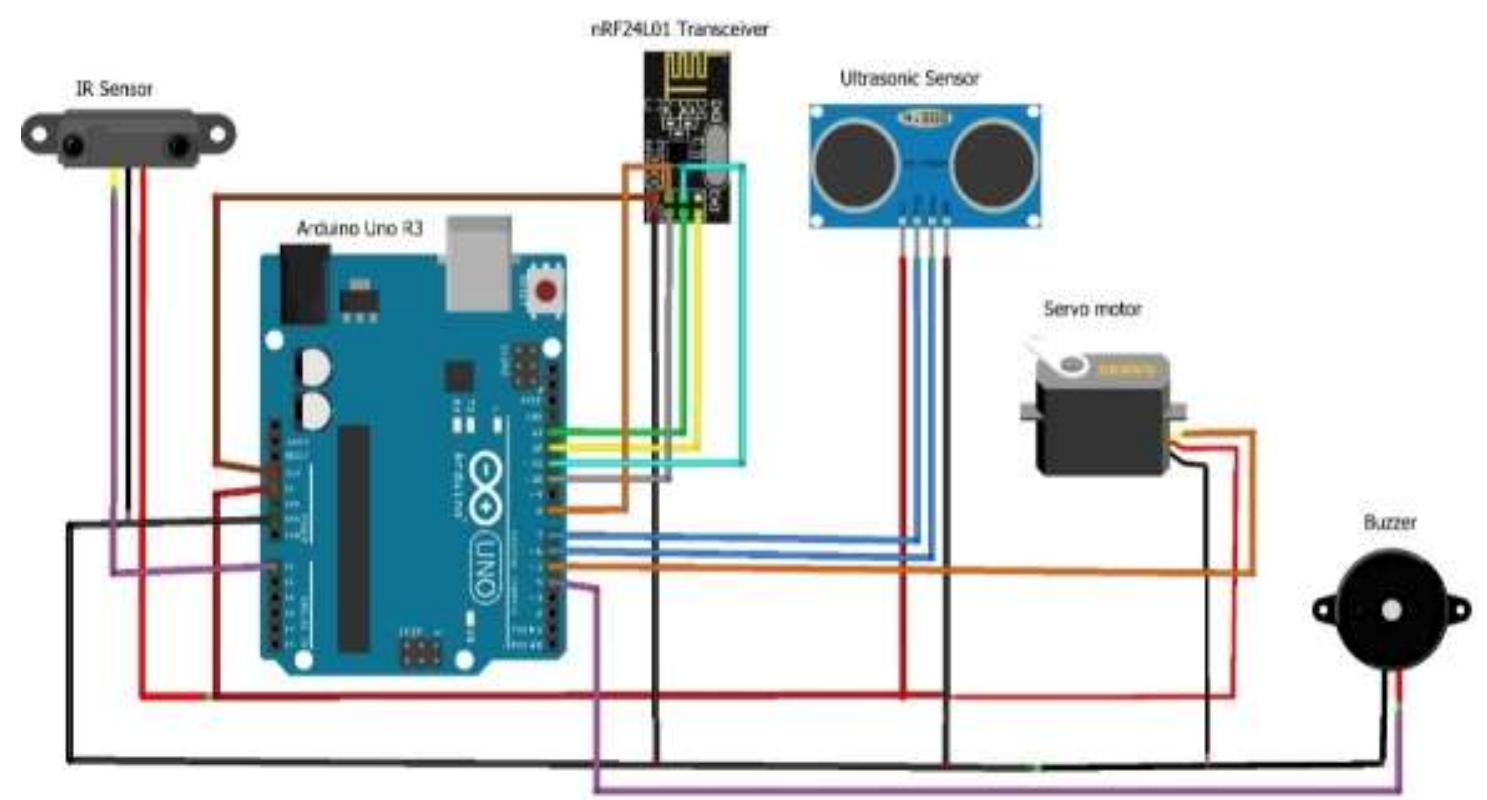

Fig 11: Implementation of an anti-collision system

\section{CONCLUSION}

This method is capable of controlling the railway gates exactly. The circuit has been test in both direction and worked perfectly. By making use of ATMEGA 16 controller can achieve a fast response compare to existing. The implementations of this kind of systems are necessary for today's railway crossings more numbers of accidents. Automatic gate control system always provides an effective way to reduce railway accidents. This work contributes a benefit to railway department and to road users. Design was
The implementation of an anti-collision system is as show in fig 11

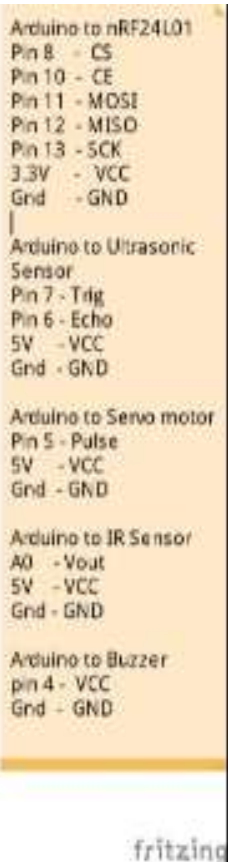

completely automated even can used in remote villages where there cannot keep station master or line man is present Two sides of gate are equipped with sensors. About arrival and departure information of the train can be obtained by this sensor. This system makes use of the servo motor to open and close the gates automatically; it is rotated clockwise or anticlockwise direction. The LCD display used to displays the information of the railway gate control system. This

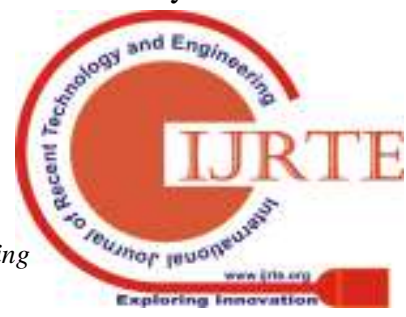


system also gives the information inters of buzzer and light indicators when train crossing.

\section{REFERENCES}

1. Duraishanmugapriyan, "Automatic railway gate controlling system" 2nd International Conference on Emerging Trends in Engineering, 20th may 2017.

2. DhanashreeAnantUmbarkar, KhushabuTalele, SamrudhiSalunke, GeetaSalunke"PLC Based Fully Automated Railway System", International Journal of Advance Engineering and Research Development, June $-2017$.

3. KarthikKrishnamurthi, Monica Bobby, Vidya v, Edwin Baby developed the "Concept of Sensor based automatic control of railway gates.

4. Acy M. Kottalil, Abhijith S, Ajmal M M, Abhilash L J,AjithBabu developed the concept of "Automatic Railway Gate Control System".

5. Md. Reya Shad Azim, Khizir Mahmud and C. K. Das developed the concept of "Automatic Train Track Switching System with Computerized Control from the Central Monitoring Unit".

6. Dr. K.R.R. Mohan Rao, SaitejaKanneganti, Chandra VenkataSaiRohit, CharanTejaSomepalli, Kantamneni Ravi Teja developed the concept of "Collision Avoidance of Trains Using Arm7".

7. RanuDewangan, PratibhadeviUmesh, "Automatic Accident Control System on Railway Tracks",

8. Dogan Ibrahim, "Smart Train Collision Detection System using a Microcontroller'International Journal of Computer Applications (0975 - 8887), October 2016.

9. Naga Hema Kumari .V, China Appala Naidu, "Train Collision Avoidance by Using Sensors"International Journal of Advanced Research in Computer Science and Software Engineering Volume 6, Issue 6, June 2016.

10. Abid Rahim, Zeeshan Ali, RaushanBharti\& Syed Sabeel N.S.P developed the concept of "Design and Implementation of a Low-Cost Wireless Sensor Network using Arduino and nRF24L01". 